\title{
Investigation of concrete by means of micro-XRF
}

\author{
Andrew Menzies, Max Buegler, Roald Tagle and Falk Reinhardt
}

Bruker Nano GmbH, Berlin, Berlin, Germany

Concrete is an important material throughout history and has been used as a building material since the Mesopotamian ages. It became very popular with Greek and Roman builders. In addition, concrete can withstand immense pressure and has a high durability, it can be poured into almost any shape, and if reinforced with steel cable or mesh, it can bridge wide distances. However, concrete does weather with time. Under the right conditions, it can last thousands of years, nevertheless, as concrete is a mixture of many complex chemical compounds, its durability depends on environmental conditions. Saltwater, for example, leads to chemical reactions that can change the volume and lead to stress and strain in the concrete structure. Important reactions in understanding concrete weathering are the Alkali-Silica Reaction (ASR) and the Alkali-Carbonate Reaction (ACR), which are described elsewhere in detail1. Such reactions can cause the concrete to expand or contract and deform, depending on the materials within the concrete.

Figure 1 is an image of the concrete sample, showing the area of analysis. The sample was analysed using micro-XRF (M4 TORNADO) under the following conditions: An area of $56.4 \mathrm{~mm}$ by $10.8 \mathrm{~mm}$, $20 \mu \mathrm{m}$ pixel size, and $10 \mathrm{~ms}$ pixel time utilizing $2 \times 30 \mathrm{~mm}^{2}$ SDDs and a Rhodium anode, with a total measurement time of 4 hours. Figure 1 is a composite elemental intensity image of this area where the gradient in $\mathrm{Cl}$ distribution (orange) is clearly visible. Chlorine $(\mathrm{Cl})$ is one of the most important elements to understand the aging and deterioration of modern concrete surfaces, as an increase in $\mathrm{Cl}$ leads to an expansion of the matrix, forming cracks and resulting in a loss of stability in any construction over time. Consequently, it is desirable to detect even small amounts of $\mathrm{Cl}$ in the matrix. It is possible to create a false color representation of the chlorine concentration to enhance the dynamic for small changes to the human eye and thus the chemical gradient of $\mathrm{Cl}$ is clearly visible by the color changing accordingly to the element's fluorescence intensity (Figure 1). With the drawing tool based on the visualization, a line has been drawn (Figure 1) and is $11.5 \mathrm{~mm}$ long, which gives an indication of the depth of $\mathrm{Cl}$ diffusion into the concrete. Additionally, the line was widened to average points perpendicular to the line. The $\mathrm{Cl}$ signal is clearly higher in the first $\sim 11.5 \mathrm{~mm}$ (from the left), and the strong modulation is a result of the large agglomerates in the matrix. Furthermore, it is possible to subdivide the map into objects with a width of $2 \mathrm{~mm}$ and almost the full height of the analysed area perpendicular to the $\mathrm{Cl}$ diffusion, and next to each other, creating individual maps that can be investigated and quantified independently. The individual maps can be analysed by phase analysis, allowing to select the integral cement phase based on characteristic elements, and thus allowing for improved quantitative analysis.2 Alternative AMICS (Automated Mineral Identification and Classification Software) can be applied to identify material in a pixel by fingerprinting.

For most micro-XRF applications rhodium (Rh) is the most versatile and favourable anode material. However, in this case, it has the major drawback that the Rh-L-lines overlap with the Cl-Klines. Therefore, careful deconvolution of the peaks is a requirement to avoid false anomalies. Alternatively, a strong filter can be introduced to suppress the Rh-L-lines, however, this will strongly hamper the sensitivity for all lighter elements, including $\mathrm{Cl}$. Therefore, utilizing an X-ray 
source that has no peak overlap with such a critical element, and ideally provides efficient $\mathrm{x}$-ray excitation of $\mathrm{Cl}$ is highly favourable. This is the case for using silver (Ag) as an anode material, which for $\mathrm{Cl}$ avoids the need for deconvolution, or requirement for a filter (Figure 2). Thus, for the analysis of concrete and the distribution of $\mathrm{Cl}$, a silver tube offers a much more efficient and potent excitation (Figure 2). The backscattered Ag-L-line intensity does not overlap with the $\mathrm{Cl}$, but it provides a much more efficient excitation of $\mathrm{Cl}$ by the $2.82 \mathrm{keV}$ absorption edge. However, a direct comparison shows that the Ag excitation comes at a cost as well, it overlaps strongly with $\mathrm{K}$. As the Ag-L-lines are transmitted more efficiently, it provides better signal for elements with $6<\mathrm{Z}<18$, such as $\mathrm{Cl}, \mathrm{S}, \mathrm{P}$, etc. It is possible to equip the micro-XRF (M4 TORNADO) with two anode sources, but only one can be equipped with a focusing poly-capillary lens.

In conclusion: An area of up to $19 \mathrm{~cm}$ x $16 \mathrm{~cm}$ can be mapped by microXRF (M4 TORNADO), The penetration of catalysts, such as $\mathrm{NaCl}$, into the concrete can be visualized and semi-quantitatively analyzed, Micro-XRF can provide compositional and mineralogical information across a range of scales, Fundamental parameter (FP) based quantification allows for quick assessment of composition in points, Mapping of mineralogy using AMICS yields information about possible ASR and ACR reactions as well as matrix and aggregate compositions, Mapping of element intensities on a drill core sample can provide visualization of alkaline hydroxide penetration into the volume, Smart analysis of mapping allows for semi-quantitative analysis of $\mathrm{Cl}$ and $\mathrm{K}$ concentration in the matrix, Phase analysis allows for quick estimation of cement to aggregate ratio as well as for semi-quantitative analysis. 

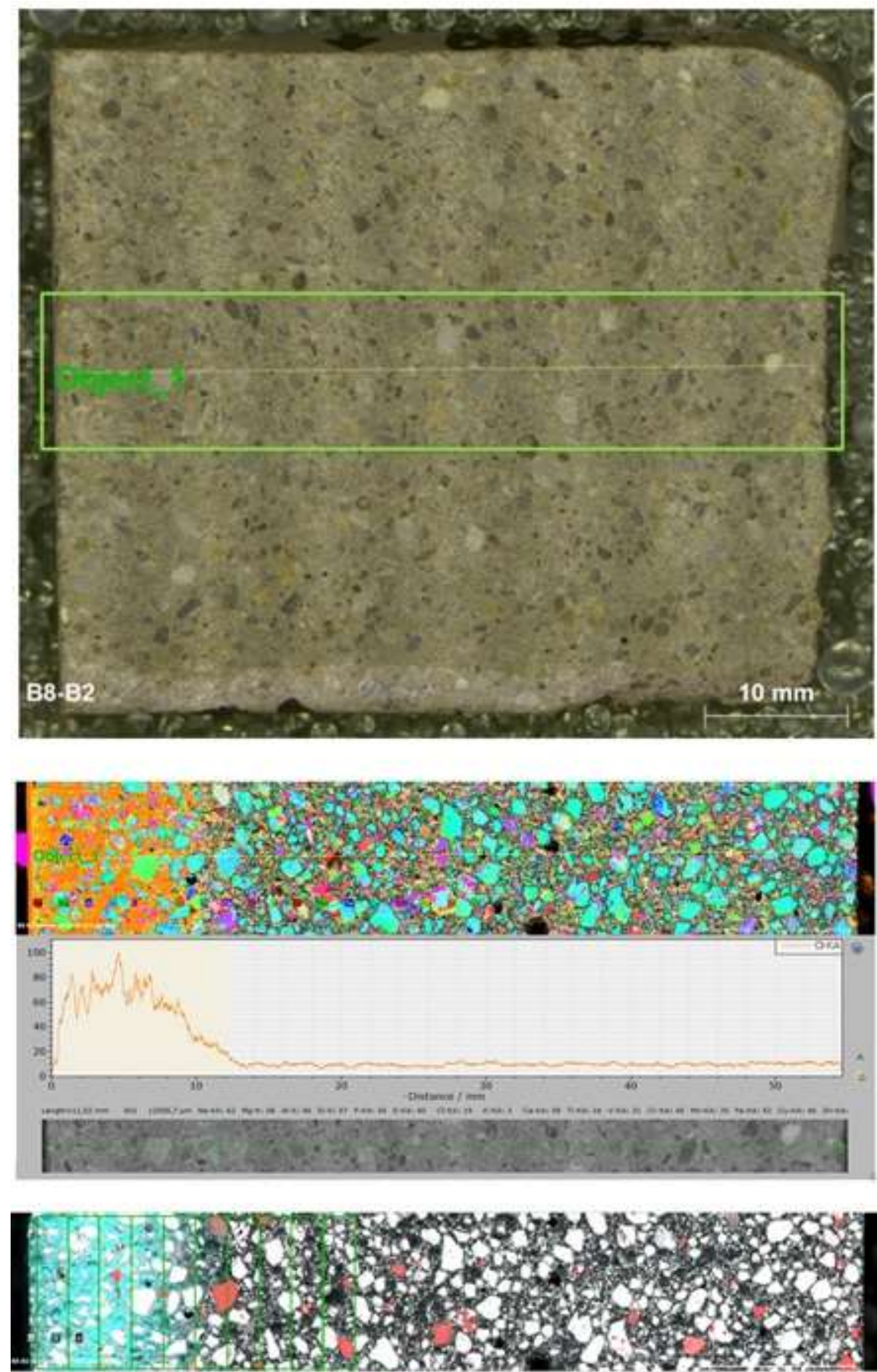

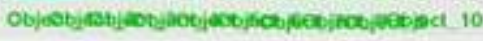
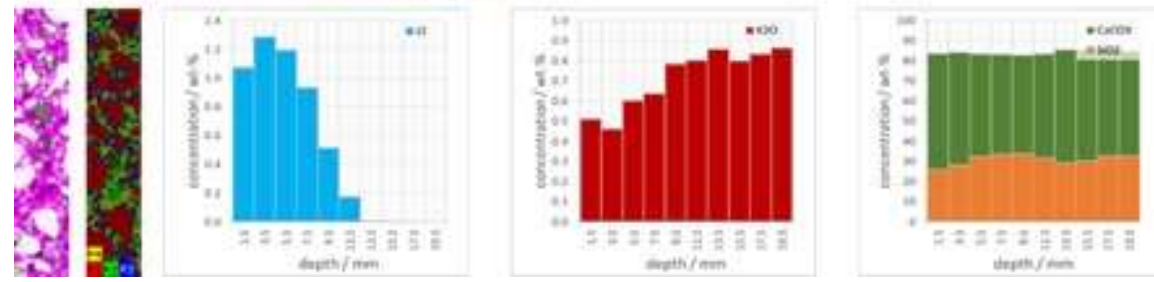

Figure 1. A stitched mosaic image from the optical camera showing the analyzed area on a concrete slab. The marked area covers $56.4 \mathrm{~mm}$ by $10.8 \mathrm{~mm}$. Multi-element composite intensity map of the area 
depicted in the top photo, with a strongly broadened line scan across the map. Beneath the resulting line profile for $\mathrm{Cl}$ along the strip is depicted. Map showing the objects, used to save separately and perform a phase analysis. Beneath the results of the quantification of the cement phase in the different bins, depending on their position with respect to the surface, are shown.

ROI of $\mathrm{Cl}$ with Rh-anode

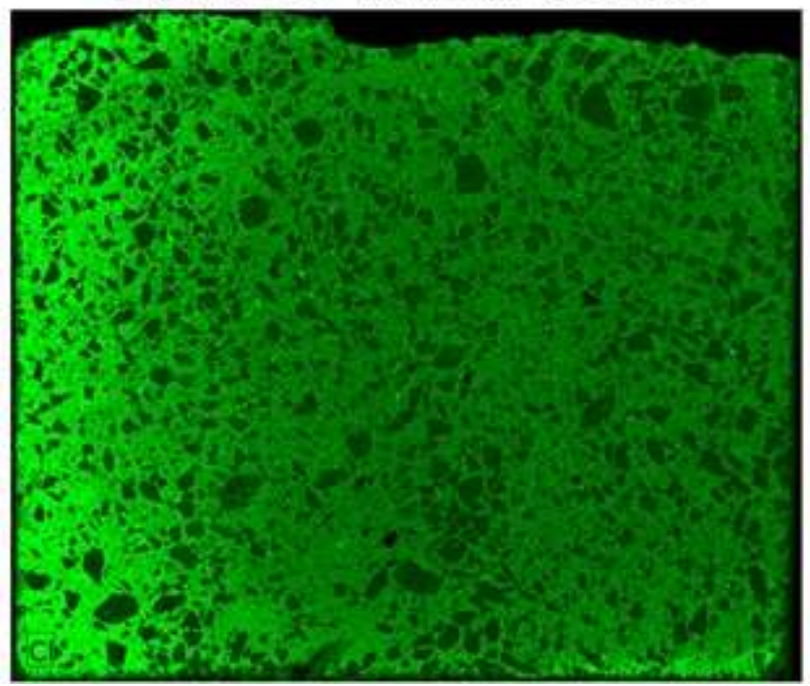

$\mathrm{ROI}$ of $\mathrm{Cl}$ with $\mathrm{Ag}$-anode

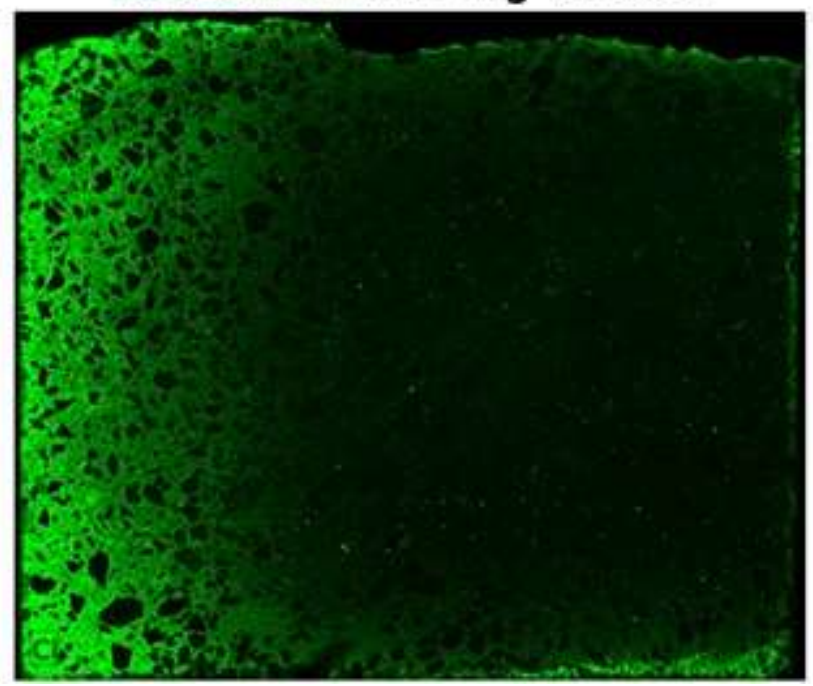

Figure 2. Chlorine $(\mathrm{Cl})$ intensity extracted from two maps of the same specimen of concrete, measured once with a Rh-anode and once with a Ag-anode. Where in the Rh excited map, there appears to be a strong $\mathrm{Cl}$ background (misinterpreted Rh-L-line intensity), there is no such artifact in the Ag excited map.

\section{References}

${ }^{1}$ F. Rajabipour, E. Giannini, C. Dunant, J. H. Ideker, M.D.A. Thomas; 'Alkali-silica reaction: Current understanding of the reaction mechanisms and the knowledge gaps; Cement and Concrete Research; Volume 76; 2015; P. 130-146; ISSN 0008-8846; https://doi.org/10.1016/j.cemconres.2015.05.024.

${ }^{2}$ J. Lindgård, T. Østnor, B. Fournier, Ø. Lindgård, T. Danner, G. Plusquellec, K. De Weerdt; 'Determining alkali leaching during accelerated ASR performance testing and in field exposed cubes using cold water extraction (CWE) and $\mu$ XRF'; 2018; DOI: 10.1051/matecconf/201819903004. 